A Trace Theorem for Solutions of

Linear Partial Differential Equations

Gang Bao

and

William W. Symes

September 1989

TR89-13 


\title{
A TRACE THEOREM FOR SOLUTIONS OF LINEAR PARTIAL DIFFERENTIAL EQUATIONS '
}

\author{
Gang Bao and William W. Symes \\ Department of Mathematical Sciences \\ Rice University \\ Houston, Texas 77251-1892 \\ U.S.A.
}

\begin{abstract}
In this paper, we prove a trace regularity theorem for the solutions of general linear partial differential equations with smooth coefficients. Our result shows that by imposing additional microlocal smoothness along certain directions. the trace of the solution on a codimension one hypersurface will be just as regular as the solution itself. The proof is based on the Hörmander-Nirenberg pseudo-differential cut-off technique and a "fattening" lemma, together with standard energy estimates.
\end{abstract}

\section{Introduction}

The standard trace theorem in Sobolev space $H^{s}\left(\mathbb{R}^{n}\right)$ indicates that the trace map which denotes the restriction of each distribution to a codimension one hypersurface extends uniquely to a continuous linear operator from $H^{s}\left(\mathbb{R}^{n}\right)$ to $H^{s-1 / 2}\left(\mathbb{R}^{n-1}\right)$, if $s>1 / 2$, see Taylor [12] or Hörmander [5] for details. It is also well known that this trace theorem is sharp. However, it seems quite natural that one may expect an improvement of regularity of the trace if the distribution is a solution of a linear partial differential equation. Obviously, the optimal case would be that of no loss (or even a gain) of smoothness. The main goal of this work is to determine circumstances under which the trace of the solution of a linear partial differential equation is as smooth as the solution itself.

\footnotetext{
'This work was partially supported by the National Science Foundation under grant DMS 86-03614 and DMS 89-05878, and by the Office of Naval Research under contracts N00014-K-85-0725 and N00014-J-89-1115.
} 
The trace properties of solutions to linear partial differential equations have been used widely in various problems such as boundary value problems, initial-boundary value problems, control problems and in particular, many inverse problems. In [9], Svmes proved a trace theorem for a second order multidimensional wave equation with constant coefficients: for finite energy initial data compactly supported away from the boundary, the trace is of class $H_{l o c}^{1}$ which is the same regularity class as the solution in the interior. His examples, in the same article, also showed that additional smoothness of initial data along certain directions (corresponding to grazing rays) is necessary for the trace to be so regular. We refer to Symes [10] for more comments, which turn out to be the original idea of our work here.

Clearly, if the linear partial differential equation is strictly hyperbolic with smooth coefficients, standard energy estimates will yield the fact that the solution along any spacelike trace is as smooth as itself locally, provided a sufficiently smooth right-hand side. Unfortunately, for more general equations or even a strictly hyperbolic differential equation but this time along a nonspacelike trace. the same idea will not work, essentially because one does not know how to apply energy estimates to a nonhyperbolic problem directly.

In this paper, we shall investigate the trace regularity of solutions to linear p.d.e. Our result shows that the difficulties discussed above may be cured by imposing some additional microlocal smoothness. In order to see why this is so, let us begin with the following definition.

Definition. A distribution $u$ is said to be in $H^{s} \cap H_{m l}^{r}\left(x_{0}, \xi_{0}\right)$ if there exist $\phi \in C_{0}^{\infty}\left(\mathbb{R}^{n}\right)$ with $\phi\left(x_{0}\right) \neq 0$ and a conic neighborhood $\Gamma \subset \mathbb{R}^{n} \backslash\{0\}$ of $\xi_{0}$ such that

$$
\langle\xi\rangle^{s}(\phi u)^{\wedge}(\xi) \in L^{2}\left(\mathbb{R}^{n}\right) \text { and }\langle\xi\rangle^{r} \chi \Gamma(\xi)(o u)^{\wedge}(\xi) \in L^{2}\left(\mathbb{R}^{n}\right) \text {. }
$$

The reader is referred to Beals [2]. Rauch [8] and references cited there for an overlook of microlocal analysis and its applications to nonlinear problems.

Roughly speaking, our trace theorem says that the solution will belong to $H^{s}$ along a codimension one hypersurface if it belongs to $H^{s}$ in a neighborhood of the hypersurface and $H^{s+1}$ in those directions where the p.d.e is not microlocally strictly hyperbolic.

The proof of the theorem is based on a pseudodifferential cutoff technique 
and standard hyperbolic energy estimates. Similar techniques have been used by many people, see for example, Hömander [6], also Nirenberg [7]. The main idea is to alter the p.d.o. microlocally to make a nice strictly hyperbolic linear pseudo-differential equation for which the trace hypersurface is spacelike, then estimate the remainder via a lemma stated in a fairly general form.

We shall also generalize our idea in the proof of the trace theorem to obtain trace regularity results to the cases where either the solution defined only on one side of the hypersurface or the trace hypersurface is characteristic.

We believe that the trace theorem, the lemma and the techniques used in this work will be helpful in various of other contexts. Some of the applications have already been seen in our recent work of understanding multidimensional hyperbolic inverse problems with smooth or nonsmooth coefficients, which will be reported elsewhere.

Notation. Throughout this paper, the reader is assumed to be familiar with the basic calculus of pseudodifferential operators ( $\psi$.d.o.) as stated in Taylor [12] or Nirenberg [7]. For simplicity, $C$ serves as a generalized positive constant the precise value of which is not needed. Usually, the constant from Fourier transform is assumed to be absorbed by the integral. $E S(P)$ stands for the essential support of operator $P$, both $\mathcal{F}$ and $\wedge$ mean Fourier transform and $\langle\xi\rangle$ is $\left(1+|\xi|^{2}\right)^{1 / 2}$.

\section{Preliminary Results}

It is interesting to see that from the definition a smooth family of $\psi$.d.o. $P\left(x, y, D_{x}\right) \in O P S_{1.0}^{r}\left(\mathbb{R}^{m}\right)$, for each $y \in \mathbb{R}^{\mathrm{n}-\mathrm{m}}$ with $m<n$, is not necessarily a $\psi$.d.o. in $\mathbb{R}^{n}$. For convenience, in the future, we shall denote the smooth family of 4 .d.o. as $P \in C^{\infty}\left(\mathbb{R}^{\mathrm{n}-\mathrm{m}}, \mathrm{OPS}_{1.0}^{\mathrm{r}}\left(\mathbb{R}^{\mathrm{m}}\right)\right)$. The results in this section will conclude that a smooth family of $\iota$.d.o. in fact behaves like a $\psi$.d.o. hence will be called a $\psi$.d.o.-like operator.

This section is devated to the understanding of these $\psi$.d.o.-like operators.

The simplest example of a smooth family of $\psi$.d.o. is a $\psi$.d.o. in fewer variables, i.e. $P\left(x, y, D_{x}\right)=P\left(x, D_{x}\right)$. Proposition 1 shows that such operators have the same Sobolev space continuity properties as ordinary $\psi$.d.o.s.

Proposition 1 If $p(x, \xi) \in S_{1,0}^{r}\left(\mathbb{R}^{m}\right), 1 \leq m \leq n$, satisfies one of the following assumptions: 
1. $p(x, \xi)=p(\xi)$, i.e., it is independent of $x$;

2. $p(x, \xi)$ has compact support in $x$,

then

$$
P\left(x, D_{x}\right): H^{s}\left(\mathbb{R}^{n}\right) \rightarrow H^{s-r}\left(\mathbb{R}^{n}\right)
$$

continuously.

Proof. For simplicity, we only prove the second statement here. The first one follows from the fact $\mathcal{F}_{x}\left[P\left(D_{x}\right) u(x, y)\right]=P(\xi) \hat{u}(\xi, y)$. It suffices to prove for $r=0$ case and derive the appropriate norm estimates. Let $u \in S$, the Schwar $z$ space, write $p(x, \xi)=\int \mathcal{F}_{x} p(\eta, \xi) e^{i x \eta} d \eta$, with $\mathcal{F}_{x} p(\eta, \xi)=\int p\left(x_{1}, \xi\right) e^{-i x_{1} \eta} d x_{1}$. Assumption 2 on $p(x, \xi)$ implies that $\left|\mathcal{F}_{x} p(\eta, \xi)\right| \leq C_{N}\langle\eta\rangle^{-N}, \forall N>0$.

$$
\mathcal{F}\left(P\left(x, D_{x}\right) u\right)(\eta, \zeta)=\int \mathcal{F}_{x} p(\eta-\xi, \xi) \hat{u}(\xi, \zeta) d \xi
$$

we have

$$
\left|\mathcal{F}\left(P\left(x, D_{x}\right) u\right)(\eta \cdot \zeta)\right| \leq C_{N} \int\langle\eta-\xi\rangle^{-N}|\hat{u}(\xi, \zeta)| d \xi
$$

Therefore,

$\left\|P\left(x, D_{x}\right) u\right\|_{H^{2}}^{2}$

$$
\leq C \int\left\|\int\langle\eta-\xi\rangle^{-(\cdot v-s)}\langle\xi, \zeta\rangle^{s}|\hat{u}(\xi, \zeta)| d \xi\right\|_{L^{2}(\eta)}^{2} d \zeta
$$

for large $N$, Young's inequality yields that

$$
\left\|P\left(x, D_{x}\right) u\right\|_{H^{s}}^{2} \leq C \int\left\|\langle\xi, \zeta\rangle^{s} \mid \hat{u}(\xi, \zeta)\right\|_{L^{2}(\xi)}^{2} d \zeta=C\|u\|_{H^{s}}^{2} .
$$

Note that, the only thing prevents $P\left(x, y, D_{x}\right)$ from being a $\psi . d . o$. of order $r$ is that its symbol $p(x, y, \xi)$ does not decrease in any directions other than $\xi$-direction. This implies that via a pseudodifferential cutoff along those nondecay directions $P$ may be regularized to be a $\psi$.d.o. which leads to our next proposition.

From now on, $\Pi_{2}: X \in T^{*}\left(\mathbb{R}^{n}\right) \rightarrow Y \in \mathbb{R}^{n} \times \mathbb{R}^{m}$ serves as a map for $n>m$,

$$
\Pi_{2}(X)=\{(x, y, \xi) \in Y:(x, y, \xi, \eta) \in X\} .
$$

Recall that the normal bundle of a foliation $\mathbb{R}^{\mathrm{n}}=\mathbb{R}^{\mathrm{n}-\mathrm{m}} \times \mathbb{R}^{\mathrm{m}}$ is the set

$$
\mathcal{N}=\left\{(x, y, \xi, \eta) \in \mathbb{R}^{\mathrm{m}} \times \mathbb{R}^{\mathrm{n}-\mathrm{m}} \times \mathbb{R}^{\mathrm{m}} \times \mathbb{R}^{\mathrm{n}-\mathrm{m}}, \xi=0\right\} .
$$


Proposition 2 Assume that $P(x, y, \xi) \in C^{\infty}\left(\mathbb{R}^{\mathbf{n}-\mathbf{m}}, \mathrm{OPS}_{1,0}^{\mathrm{r}}\left(\mathbb{R}^{\mathrm{m}}\right)\right), H\left(x, y, D_{x}, D_{y}\right) \in$ $O P S_{1,0}^{s}\left(\mathbb{R}^{n}\right), 1 \leq m \leq n, r, s \in \mathbb{R}$ and $\phi(x, y) \in C_{0}^{\infty}\left(\mathbb{R}^{n}\right)$. Furthermore, assume that

$$
E S(H) \cap \mathcal{N}=\emptyset,
$$

where $\mathcal{N}$ is the normal bundle of $\mathbb{R}^{\mathrm{m}} \times \mathbb{R}^{\mathrm{n}-\mathrm{m}}$. Then

$$
P \phi H \in O P S_{1,0}^{r+s}\left(\mathbb{R}^{n} \times \mathbb{R}^{n}\right)
$$

and

$$
E S(P \phi H) \subset \Pi_{2} E S(P(\cdot, y, \cdot)) \cap E S(H) .
$$

Proof. W.L.O.G., it is sufficient to consider the case with $r=s=0$ (some simple modifications lead to the general case). Thus it suffices to show that

$$
Q=P\left(x, y \cdot D_{x}\right) \phi(x, y) H\left(D_{x}, D_{y}\right)
$$

is a $\psi$.d.o. ( in $\left.O P S_{1,0}^{0}\left(\mathbb{R}^{n} \times \mathbb{R}^{n}\right)\right)$. Observe that $Q u(x, y)$

$$
=\iint\left[\int P(x, y, \xi) \hat{o}\left(\xi-\xi^{\prime} \cdot y\right) \epsilon^{i x\left(\xi-\xi^{\prime}\right)} d \xi\right] H\left(\xi^{\prime}, \eta^{\prime}\right) \hat{u}\left(\xi^{\prime} \cdot \eta^{\prime}\right) e^{i x \xi^{\prime}+i y \eta^{\prime}} d \xi^{\prime} d \eta^{\prime},
$$

hence, the symbol of $Q$

$$
Q_{0}=\int P\left(x, y, \xi+\xi^{\prime}\right) \hat{\phi}(\xi, y) e^{i x \xi} d \xi H\left(\xi^{\prime}, \eta^{\prime}\right) .
$$

The definition of $\psi$.d.o. gives that

$$
\begin{aligned}
\left|\partial_{\xi^{\prime}}^{\alpha_{1}} P\left(x, y, \xi+\xi^{\prime}\right)\right| & \leq C_{\alpha, K^{\prime}}\left(1+\left|\xi+\xi^{\prime}\right|\right)^{-\alpha_{1}} \\
& \leq C\left(1+\left|\xi^{\prime}\right|\right)^{-\alpha_{1}}(1+|\xi|)^{\alpha_{1}}, \forall \alpha_{1}>0 .
\end{aligned}
$$

therefore, for any $\left(\xi^{\prime}, \eta^{\prime}\right) \in \mathbb{R}^{n}$ and $(x, y) \in K^{\prime}$ (a compact set in $\left.\mathbb{R}^{n}\right)$, $\left|\partial_{\xi^{\prime}}^{\alpha} Q_{0}\left(x, y, \xi^{\prime}, \eta^{\prime}\right)\right|$

$$
\begin{aligned}
& \leq C\left|\sum_{0 \leq \alpha_{1} \leq \alpha}\left[\partial_{\xi^{\prime}}^{\alpha_{1}} \int P\left(x, y \cdot \xi+\xi^{\prime}\right) e^{i x \xi} \hat{\phi}(\xi, y) d \xi\right] \partial_{\xi^{\prime}}^{\alpha-\alpha_{1}} H\left(\xi^{\prime}, \eta^{\prime}\right)\right| \\
& \leq \sum_{0 \leq \alpha_{1} \leq \alpha} C\left(1+\left|\xi^{\prime}\right|\right)^{-\alpha_{1}} \partial_{\xi^{\prime}}^{\alpha^{-}-\alpha_{1}} H\left(\xi^{\prime} \cdot \eta^{\prime}\right) \\
& \leq C_{\alpha, K}\left(1+\left|\xi^{\prime}\right|+\left|\eta^{\prime}\right|\right)^{-\alpha} .
\end{aligned}
$$


where the last inequality comes from our construction of $H$, i.e. $H\left(\xi^{\prime}, \eta^{\prime}\right)$ nonzero only in the region $\left(1+\left|\xi^{\prime}\right|+\left|\eta^{\prime}\right|\right) \leq C\left(1+\left|\xi^{\prime}\right|\right)$.

The fact

$$
E S(P \phi H) \subset \Pi_{2}^{-1} E S(P(\cdot, y, \cdot))
$$

is a simple exercise of the definition of essential support as well as the map $P_{2}$. From the above expression of the symbol of $Q$, it is obvious to see that

$$
E S(P \phi H) \subset E S(H)
$$

Remark. In the appendix of [12], Taylor studied some properties of $\psi$.d.o.like operators through two lemmas (Lemma A.1 and Lemma A.2). While in Lemma A.1, for a smooth family of $\psi$.d.o. $P_{1} \in C^{\infty}\left(\mathbb{R}^{1}, \mathrm{OPS}_{1,0}^{1}\left(\mathbb{R}^{\mathbf{n}-1}\right)\right)$, he obtained essentially the first conclusion of Proposition 3.2. He then showed in Lemma A.2 that if $\left(\partial / \partial_{y}-P_{1}\right) u \in C^{\infty}$, then $W F(u) \cap \mathcal{N}=\emptyset$.

We shall make an extensive use of the $\psi$.d.o. cut-off technique behind Proposition 2 and examine further properties of these $\psi$.d.o.-like operators.

\section{Trace Theorem}

We can now state and prove the main result in this paper, a trace theorem. From now on. $t$ will serve as a distinguished variable. For $m>n, \Pi^{-1}$ : $X \in T^{*}\left(\mathbb{R}^{n}\right) \rightarrow Y \in T^{*}\left(\mathbb{R}^{m}\right)$ denotes the puliback map, i.e. $\Pi^{-1}\left(X^{-}\right)=$ $\{(x, y, \xi, \eta) \in Y, \quad(x, \xi) \in X\}$.

Theorem. Assume that

$$
P(x, t, \xi, \tau)=\tau^{m}+\sum_{j=0}^{m-1} a_{\jmath}(x, t) \tau^{\jmath} \xi^{m-\jmath}
$$

is the principal symbol of a linear partial differential operator $P\left(x, t, D_{x}, D_{t}\right)$ with uniformly bounded smooth coefficients $\left\{a_{j}(x, t)\right\}$.

Let $\Omega \subset \subset \mathbb{R}^{n}=\{t=0\}$ and $\gamma=$ closed conic set $\left.\subset T^{*}\left(\mathbb{R}^{n}\right)\right|_{\bar{\Omega}}$ such that $(x, \xi) \in \gamma \Rightarrow P(x, 0, \xi, \tau)$ has $m$ distinct real roots as a polynomial of $\tau$.

Let $\Gamma$ be a closed conic set which does not intersect the normal bundle of $\{t=0\}$. Also, assume that $u$ satisfies the equation $P u=0$ and

$$
u \in H^{s} \cap H_{m l}^{s+1}\left(\Gamma \cap \gamma^{\prime}\right),
$$


where $\gamma^{\prime}=\Pi^{-1}\left(\gamma_{1}\right) \subset T^{*}\left(\mathbb{R}^{n+1}\right)$ and $\gamma_{1}$ is a conic neighborhood of $\gamma^{C}$. Then, for $\phi(x, t) \in C_{0}^{\infty}\left(\Omega_{1}\right)$ with $\Omega_{1} \subset \subset \mathbb{R}^{n+1},\left.\Omega_{1}\right|_{t=0} \subset \Omega$,

$$
\left.\phi u\right|_{t=0} \in H^{s}
$$

Remarks. If $P$ is elliptic, the stronger conclusion holds by the classical trace theorem. On the other hand, if $P$ is strictly hyperbolic with respect to the trace $\{t=0\}$, the conclusion also holds from standard hyperbolic energy estimates. Moreover, the conclusion of the theorem can not be improved significantly if the operator is not elliptic.

The equation is not necessarily homogeneous. Also, there are no more difficulties if the operator has smooth lower order terms. In many situations, the microlocal smoothness assumption of the solution can be imposed by appropriate side conditions, such as Cauchy data for hyperbolic problems.

Although in this paper only the p.d.e. with smooth coefficients are considered, we claim that it is entirely possible to prove trace theorems for general nonsmooth coefficients cases by analyzing various results on linear propagation of singularities, for example those in Beals and Reed [3] and [4], on this matter partial results have be obtained in Bao [1].

Proof of Theorem. For the situations where the operator $P$ is neither elliptic nor strictly hyperbolic, i.e. both $\gamma$ and $\gamma^{C}$ are not empty, the idea of proof is to construct a strictly hyperbolic Cauchy problem by a $\psi$.d.o. cutoff. Since the problem is local, one may assume that $\gamma=\Omega \times \mathcal{U}$ and $\gamma_{1}^{C}=\Omega \times \mathcal{U}_{1}$. where $\mathcal{U}$ is a conic neighborhood of $\mathcal{U}_{1}$. which guarantee that we can find a convolutional w.d.o. $Q \in O P S_{1,0}^{0}\left(\mathbb{R}^{n} \times \mathbb{R}^{n}\right)$ (i.e. $\left.Q(x . \xi)=Q(\xi)\right)$ such that

- $E S(Q) \subset \mathcal{U}$

- $Q_{0}=1$ in $\mathcal{U}_{1}$ and $0 \leq Q_{0} \leq 1$,

where $Q_{0}$ is the principal symbol of $Q$.

Observe that

$$
Q P=Q \prod_{i=1}^{m_{2}}\left(\tau-k_{i}(t \cdot r . \xi)\right)
$$

where $\left\{k_{i}\right\}$ are real and distinct.

The characteristic set $C$ in $\Pi^{-1}(E S(Q))$ is the union of $m$ conic sets

$$
C^{(i)}=\left\{(t, x, \tau, \xi) \neq 0: \tau=k_{i}\right\} \cap \Pi^{-1}(E S(Q)) .
$$


Following the idea in Nirenberg [7] or Beals and Reed [3], a simple partition of unity argument allows one to choose $\left\{b_{i}\right\}, R \in O P S_{1,0}^{0}$ such that: (1) $b_{i}$ elliptic in a small conic nbhd of $C^{(i)}$ and supp $b_{i} \cap\left(\cup_{j \neq i} C^{(j)}\right)=\emptyset ;(2) R$ is properly supported and $\operatorname{supp} R \cap\left(\cup_{3} C^{(j)}\right)=\emptyset ;(3) R+\sum_{2} b_{i}=I$. Then, by the calculus of $\psi$.d.o. we can find $q_{m-1}^{(i)} \in O P S_{1,0}^{m-1}$ elliptic near $C^{(2)}$ with

$$
P b_{i}=\left(D_{t}-k_{i}\right) q_{m-1}^{(i)} b_{i} \text { (modulo smoothing operators). }
$$

Define, for $k_{i}^{0}$ real number, that

$$
\tilde{P}_{i}=Q\left(D_{t}-k_{i}\right)+(I-Q)\left(D_{t}-k_{i}^{0}|\xi|\right) .
$$

It follows that $\tilde{P}_{i}$ is strictly hyperbolic $\psi$.d.o. of order one and differential in $t$,

$$
\dot{P}_{i} q_{m-1}^{(i)} b_{i} r_{i} \phi u=Q P b_{i} r_{i} \phi u+(I-Q)\left(D_{t}-k_{i}^{0}|\xi|\right) q_{m-1}^{(i)} b_{i} r_{i} \phi u,
$$

with $r_{i} \in O P S_{1,0}^{m-1}$ is the parametrix of $q_{m-1}^{(i)}$ near $C^{(i)}$. Standard hyperbolic energy estimates and pseudolocal property of $\psi$.d.o. (see, e.g. Taylor [12]) as well as Proposition 1 yield

$$
\left\|\left.\left(b_{i} \phi u\right)\right|_{t=0}\right\|_{s} \leq C\left[\left\|Q\left[P, b_{i} r_{i} \phi\right] u\right\|_{s}+\left\|(I-Q) b_{i} \phi u\right\|_{s+1}\right],
$$

hence, there is a $\varphi_{0} \in C_{0}^{x}$.

$$
\left\|\left.\left(\sum b_{i} \phi u\right)\right|_{t=0}\right\|_{s} \leq C\left[\left\|o_{0} u\right\|_{s}+\|(I-Q) \phi u\|_{s+1}\right] .
$$

On the other hand, it is clear that $P$ is elliptic in $\Pi^{-1}(E S(Q)) \backslash \cup_{j} C^{(j)}$, thus $R Q \phi u \in H^{s+1}$.

Combining the above arguments, we obtain that II (ou) $\left.\right|_{t=0} \|_{s}$ $\leq C\left[\left\|\phi_{0} u\right\|_{s}+\|(I-Q) \phi u\|_{s+1}+\left\|\left.(R Q \phi u)\right|_{t=0}\right\|_{s}+\left\|\left.(R(I-Q) \phi u)\right|_{t=0}\right\|_{s}\right]$. The standard trace theorem and Proposition 1 give

$$
\left\|\left.(\phi u)\right|_{t=0}\right\|_{s} \leq C\left[\left\|\phi_{0} u\right\|_{s}+\|(I-Q) \phi u\|_{s+1}+\|R Q O u\|_{s+1}\right] .
$$

In order to complete our proof it suffices to show that

$$
(I-Q) \oplus u \in H^{s+1}\left(\mathbb{R}^{n+1}\right),
$$


which requires the following lemma.

Lemma. Let $B\left(x, y, D_{x}\right) \in C^{\infty}\left(\mathbb{R}^{\mathrm{n}_{0}-\mathrm{m}_{0}}, \mathrm{OPS}_{1,0}^{\mathrm{r}}\left(\mathbb{R}^{\mathrm{m}_{0}}\right)\right.$ and $A\left(x, y, D_{x}, D_{y}\right) \in$ OPS $S_{1,0}^{s}\left(\mathbb{R}^{n_{0}}\right)$, where $1 \leq m_{0} \leq n_{0}$. Let

$$
\mathcal{N}=\left\{(x, \xi) \in \mathbb{R}^{\mathrm{n}_{0}} \times \mathbb{R}^{\mathrm{n}_{0}},\left(\xi_{1}, \cdots, \xi_{\mathrm{m}_{0}}\right)=0\right\}
$$

be the normal bundle of $\mathbb{R}^{\mathrm{m}_{0}} \times \mathbb{R}^{\mathrm{n}_{0}-\mathrm{m}_{0}}$. Also, assume that

1. A is microlocal elliptic on a conic set $\operatorname{Ell}(A)$, with $\mathcal{N} \subset \subset \operatorname{Ell}(A)$;

2. $u \in H^{l} \cap H_{m !}^{l+1}\left(\left[T^{*}\left(\mathbb{R}^{\mathrm{n}_{0}}\right) \backslash \operatorname{Ell}(\mathrm{A})\right] \cap \Pi_{2}^{-1} \operatorname{ES}(\mathrm{B}(\cdot, y, \cdot))\right)$;

3. $A \phi u \in H_{l o c}^{l-s+1}\left(\mathbb{R}^{n_{0}}\right)$. where $\phi(x) \in C_{0}^{\infty}\left(\mathbb{R}^{n_{0}}\right)$.

Then

$$
\text { Bou } \in H_{l o c}^{l-r+1}\left(\mathbb{R}^{n_{0}}\right) \text {, }
$$

in addition, if $B$ is either a convolutional operator or it symbol has compact support in space variables,

$$
B \phi u \in H^{l-r+1}\left(\mathbb{R}^{n_{0}}\right) .
$$

To be able to apply the above lemma to our proof of the trace theorem, the assumptions stated in the lemma must be rerifed. Assumption 2 is just our assumption in the theorem. Assumption 3 is easy to be verified. Since the coefficients $\left\{a_{j}(x, t)\right\}$ are uniformly bounded. $P(x, t . \xi . \tau)$ will be microlocal elliptic in a conic neighborhood $\mathcal{K}$ of $\mathbb{R}^{2(n+1)} \backslash \Gamma$ if $\Gamma$ is properly chosen, this verifies the assumption 1 of the lenma. Thus, as an immediate consequence of the lemma, we obtain that

$$
(I-Q) \phi u \in H^{s+1}
$$

which completes the proof of our trace theorem.

Remark on the Lemma. The operator A plays a very important role here. Fortunately, for many problems the operator in the linear partial differential equation can be often chosen as A, which is implied by assumption 3.

Proof of Lemma. We only prove the first part of the conclusion, the second part can be shown by a simple application of our proposition 1 .

By assumptions 1 and 2 , we can find a conic set $E l_{1}$, such that $\mathcal{N} \subset$ $E l l_{1} \subset \subset E l l(A)$, and $u \in H^{l} \cap H_{m l}^{l+1}\left(\left[T^{*}\left(\mathbb{R}^{\mathrm{n}_{0}}\right) \backslash \mathrm{E} \|_{1}\right] \cap \Pi_{2}^{-1} \operatorname{ES}(\mathrm{B}(\cdot, y, \cdot))\right)$.

One can also construct a $\psi$. d.o. $H_{1} \in O P S_{1,0}^{0}\left(\mathbb{R}^{\mathrm{n}_{0}}\right)$ which satisfies 
- $E S\left(H_{1}\right) \subseteq E l l(A)$ and

- the symbol of $H_{1}, h_{1}=1$ on $E l l_{1} \cap\{(x, \xi):|\xi|>1\}$.

Write $\phi=\phi \phi_{1}$ with $\phi_{1} \in C_{0}^{\infty}\left(\mathbb{R}^{n_{0}}\right)$; we then have

$$
B \phi u=B \phi_{1} H \phi u+B \phi_{1}(I-H) \phi u
$$

with $H=I-H_{1}$.

Since

$$
A H_{1} \phi u=\left[A, H_{1}\right] \phi u+H_{1} A \phi u
$$

and $\left[A, H_{1}\right]$ has order $s-1$, we have

$$
A H_{1} \propto u \in H_{l o c}^{l-s+1}
$$

which follows by assumptions $18 \cdot 2$ and Proposition 1.

From assumption 1 and the fact

$$
E S\left(H_{1}\right) \subseteq E l l(A)
$$

where $\operatorname{Ell}(A)$ is the microlocal elliptic region of $A$, it is easy to see that

$$
H_{1} \phi u \in H_{l o c}^{l+1} \text {. }
$$

Thus Proposition 1 gives

$$
B o_{1} H_{1} \text { ou } \in H_{l o c}^{l-r+1}
$$

On the other hand, from the construction of $H$. our Proposition 2 implies that

$$
B \phi_{1} H \in O P S_{1,0}^{r}\left(\mathbb{R}^{n_{0}}\right)
$$

Moreover,

$E S\left(B \phi_{1} H\right) \subseteq \Pi_{2}^{-1} E S(B(\cdot, y, \cdot)) \cap E S(H) \subseteq\left[T^{*}\left(\mathbb{R}^{n_{0}}\right) \backslash E l_{1}\right] \cap \Pi_{2}^{-1} \operatorname{ES}(B(\cdot, y, \cdot))$.

Therefore, a simple property of wavefront set vields

$$
B \phi_{1} H \phi u \in H_{l o c}^{l+1} \text {. }
$$

Eventually, combining the above arguments, we have

$$
B \phi u=B \phi_{1} H \phi u+B \phi_{1} H_{1} \phi u \in H_{l o c}^{l+1}
$$

which finishes the proof. 


\section{Half Space and Characteristic Trace The- orems}

The aims of this section are to carry over our ideas in last sections to some more difficult situations. It is obviously crucial to investigate the trace regularity of solutions to linear partial differential equations defined only on one side of the trace hypersurface (corresponding to boundary value problems). The difficulties will present immediately due to the fact that the $\psi$.d.o. cutoff technique engaged in the proof of the previous lemma will break down around the trace in general. Nevertheless, one is able to get some partial results along the same line of the proof of the trace theorem as follows.

Corollary 1 (half space trace theorem) Assume that $P\left(x, t, D_{x}, D_{t}\right)$ is a linear partial differential operator of order $m$ with smooth coefficients. Also, assume there is a smooth family of u.d.o. and a small constant $\epsilon>0$,

$$
Q(t)=Q\left(x, t, D_{x}\right) \in C^{\infty}\left\{[0, \epsilon], O P S_{1,0}^{0}(\Omega)\right\}
$$

with $\Omega$ is an open subset of $\mathbb{R}^{n}$. such that

(1). if $(x, \xi) \in[E S(Q)]^{C}$, for all $0 \leq t \leq \epsilon, P(x, t, \xi, \tau)$ has $m$ distinct real roots as a polynomial of $\tau$ :

(2). $\left.(Q u)\right|_{t=t_{0}} \in H_{\text {loc }}^{s+1}(\Omega), 0 \leq t_{0} \leq \epsilon$.

where $u \in H_{\text {loc }}^{s}(\Omega \times[0, \epsilon])$ satisfies the equation $P u=0$ in $\Omega \times[0, \epsilon]$.

Then, for $\phi(x, t) \in C_{0}^{\infty}(\Omega \times[-\epsilon, \epsilon])$.

$$
\left.(\phi u)\right|_{t=0} \in H^{s}(\Omega) \text {. }
$$

Idea of the proof. We basically follow the proof of the trace theorem, making the necessary modifications. Let us first extend $u$ (in whatever way) to a small neighborhood of the trace such that

$$
u \in H_{l o c}^{s}(\Omega \times[-\epsilon, \epsilon]) .
$$

W.L.O.G. we may assume that $u(x, t)$ has compact support in $x$. Moreover, the pseudolocal property allows one to assume further that $Q$ is compactly supported in $x$. Therefore, for all $r \in \mathbb{R}$

$$
Q: H^{r}(\Omega) \rightarrow H^{r}(\Omega) \text {, uniformly in } t \in[0, \epsilon]
$$


Knowing the facts

$$
\int_{0}^{\epsilon}\|\phi Q u\|_{H^{s+1}(\Omega)}^{2} d t<+\infty \text { and } u \in H_{l o c}^{s}(\Omega \times[-\epsilon, \epsilon]),
$$

the same technique as in the proof of the trace theorem will yield the conclusion.

Another interesting case would be that the trace hypersurface is characteristic, then a slightly different $\psi$.d.o. cutoff technique will be needed. To clarify the ideas, we assume that. after some coordinates transformation, the characteristic hypersurface is $\{t=0\}$ and furthermore the operator has a simple form. We then have the following result.

Corollary 2 (characteristic trace theorem) Assume that $P\left(x, t, D_{x}, D_{t}\right)$ is a linear partial differential operator of order $m$ with smooth coefficients,

$$
P\left(x, t, D_{x}, D_{t}\right)=B\left(x, D_{x}\right) \partial / \partial t-A\left(x, D_{x}\right)
$$

with $A, B$ are differential operators of order $m+1, m$ respectively. Assume there is a \&.d.o. $Q\left(x, D_{x}\right) \in O P S_{1.0}^{0}(\Omega), \Omega$ is an open subset in $\mathbb{R}^{n}$, such that

(1). if $(x, \xi) \in[E S(Q)]^{C}$, then the principal part of $B(x, \xi) \neq 0$

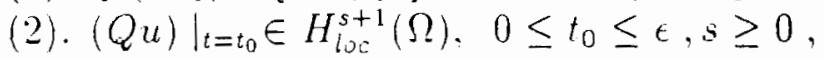
where $u \in H_{l o c}^{s}(\Omega \times[0, \epsilon])$ satisfies the equation $P u=0$ in $\Omega \times[0, \epsilon]$. Then, for $\phi(x, t) \in C_{0}^{\infty}(\Omega \times[-\epsilon, \epsilon])$.

$$
\left.(B \phi u)\right|_{t=0} \in H^{s-m}(\Omega) \text {. }
$$

Sketch of the proof. Observe that. for $P_{1}=P / B$ formally,

$$
\tilde{P}=(I-Q) P_{\mathbf{l}}+Q P_{0}
$$

with $P_{0}$ a strictly hyperbolic p.d.o. of order one. Again, $\dot{P}$ is a strictly hyperbolic $\psi$.d.o. of order one and is differential in $t$. From

$$
\check{P} B \phi u=(I-Q)[P . \phi] u+Q P_{0} B \phi u
$$

the similar arguments as before will vield the conclusion. 


\section{References}

[1] Bao, G., Microlocal Regularity of an Inverse Problem for the Multidimensional Wave Equation, Ph.D. thesis, Department of Mathematical Sciences, Rice University, Houston, Texas, 1990.

[2] Beals, M., Propagation and Interaction of Singularities in Nonlinear Hyperbolic Problems, Birkhäuser, Boston, 1989.

[3] Beals, M. and Reed, M.. Propagation of Singularities for Hyperbolic Pseudodifferential Operators and Applications to Nonlinear Problems, Comm. Pure Appl. Math. 35. 1982, 169-184.

[4] Beals, M and Reed, M.. Microlocal Regularity Theorems for Nonsmooth Pseudodifferential Operators and Applications to Nonlinear Problems, Trans. Am. Math. Soc. 285. 1984, 159-184.

[5] Hörmander, L., Linear Partial Differential Operators. Springer Verlag, New York, 1969.

[6] Hörmander, L., Linear Differential Operators. Actes Congr. Internat. Math., Vol.1 (Nice, 1970), Gauthier-Villars, Paris, 1971, 121-133.

[7] Nirenberg, L., Lectures on Linear Partial Differential Equations, CBMS Regional Conf. Ser. in Math.. no. 17. Amer. Math. Soc., Providence, R.I.. 1973.

[8] Rauch. J., Singularities of Solutions to Semilinear Wave Equations, J. Math. Pures Appl. 58, 1979, 299-308.

[9] Symes, W. W., A Trace Theorem for Solutions of the Wave Equation, and the Remote Determination of Acoustic Sources. Nath. Mech. in the Appl. Sci. 5, 1983, 131-152.

[10] Symes, W. W., Some Aspects of Inverse Problems in Several Dimensional Wave Propagation, Proc. Conference on Inverse Problems, SIAMAMS Proceedings 14, Amer. Math. Soc., Providence, R.I., 1983.

[11] Taylor, M., Reflection of Singularities of Solutions to Systems of Differential Equations, Comm. on Pure and Appl. Math. 28, 1975, $457-475$. 
[12] Taylor, M., Pseudo-Differential Operators, Princeton Univ. Press, Princeton, N.J., 1981. 